\title{
CICLOADIÇÕES [3+4] VIA CÁTIONS OXIALÍLICOS: APLICAÇÕES EM SÍNTESES ORGÂNICAS
}

Antônio Jacinto Demuner, Luiz Cláudio de Almeida Barbosa*

Departamento de Química - Universidade Federal de Viçosa - 36571-000 - Viçosa - MG

Dorila Piló-Veloso

Departamento de Química - Universidade Federal de Minas Gerais - 31270-901 - Belo Horizonte - MG

Recebido em 8/12/95; aceito em 29/8/96

\begin{abstract}
[3+4]CYCLOADDITIONS VIA OXYALLYL CATIONS: APPLICATIONS IN ORGANIC SYNTHESIS. Several methodologies for the generation of oxyallyl cations from polybromoketones and other substrates are discussed. The mechanistic aspect of the $[3+4]$ cycloaddition reaction between these cations and dienes leading to the formation of seven membered ring carbocyclic compounds is presented. Finally, some synthetic applications of the $[3+4]$ cycloaddition are shown.
\end{abstract}

Keywords: [3+4] cycloaddition; oxyallyl cation; synthesis.

1. Introdução

2. Formação e Reatividade de Cátions Oxialílicos

2.1. Obtenção de cátions oxialílicos a partir de $\alpha$-halocetonas

2.2. Obtenção de cátions oxialílicos a partir de $\alpha, \alpha^{\prime}$-dialocetonas e de $\alpha, \alpha, \alpha^{\prime}, \alpha^{\prime}$-tetraalocetonas

2.3. Obtenção de cátions oxialílicos ou alílicos a partir de outros substratos

2.4. Mecanismos de cicloadição de cátions oxialílicos com 1,3-dienos

3. Aplicações Sintéticas

3.1. Obtenção de compostos bifenílicos e de troponas

3.2. Obtenção de ozonídeos

3.3. Obtenção de alcalóides tropânicos

3.4. Estudos visando a obtenção de lactonas sesquiterpênicas

3.5. Estudos visando a obtenção de análogos oxigenados do ácido helmintospórico

3.6. Obtenção de C-nucleosídeos

3.7. Estudos visando o preparo do esqueleto taxano

4. Conclusão

\section{INTRODUÇÃO}

Compostos cíclicos são amplamente encontrados na natureza dentre os quais aqueles possuindo anéis de cinco e seis membros são os mais abundantes. Entretanto, produtos com anéis menores ou maiores que estes são de grande importância e têm constituído objeto de desenvolvimento de muitas metodologias de síntese ${ }^{1}$. Neste trabalho será apresentada uma revisão sobre a síntese de substâncias contendo anéis de sete membros, cuja metodologia envolva a formação de cátions oxialílicos e alílicos.

Diferentemente da síntese de compostos com anéis de seis membros que podiam ser obtidos pela reação de Diels-Alder, uma cicloadição [4+2], o preparo de carbocíclicos com sete membros envolvia, até 1962, reações de expansão e/ou contração de anéis ${ }^{2}$. Em 1962, Fort $^{3-5^{5}}$ descreveu um procedimento para o preparo direto de carbociclos de sete membros, através da reação de cicloadição [3+4] entre um cátion oxialílico (fonte de três carbonos) e um dieno (unidade com quatro carbonos).

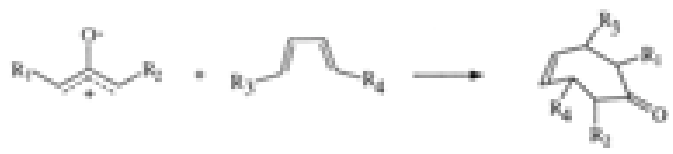

Em termos eletrônicos, de acordo com as regras de Woodward-Hoffmann, essa reação é uma cicloadição iônica do tipo $[\pi 4 s+\pi 2 s]$, uma vez que estão envolvidos 6 elétrons $\pi$ no processo. Entretanto, em termos sintéticos é classificada como cicloadição [3+4], uma vez que estão envolvidas duas espécies com 3 e 4 átomos de carbono cada.

Em seu experimento clássico, Fort ${ }^{3-5}$ tratou 1-cloro-1,3difenilpropanona (1), furano e 2,6-lutidina em DMF, obtendo uma mistura isomérica dos cicloadutos $\mathbf{2 a}$ e $\mathbf{2 b}$. Quando essa reação foi repetida em presença de metanol, obteve-se somente o produto $\mathbf{3}$, proveniente da substituição do cloro da cetona $\mathbf{1}$ (Esquema 1). Fort realizou o estudo da cinética dessa metanólise e verificou que, neste caso, a formação do composto 3 ocorre através do intermediário 4 , o qual pode ser originado a partir do cátion oxialílico $\mathbf{5}$, conforme ilustrado no esquema 2 .

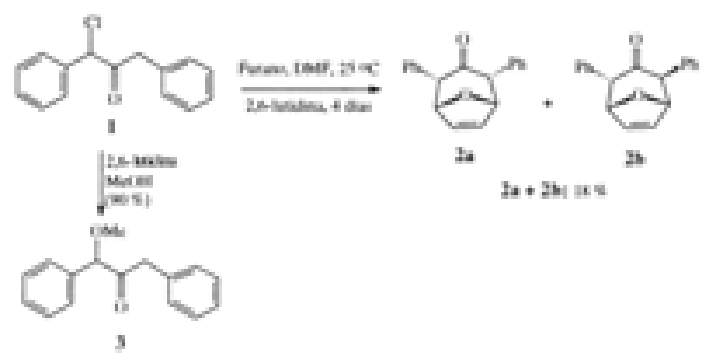

Esquema 1

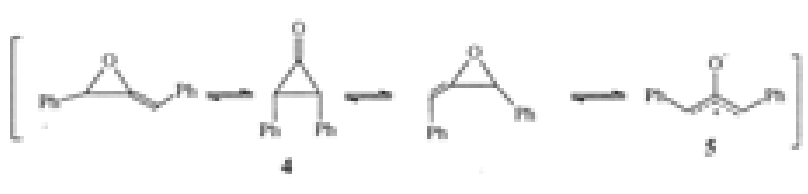

Esquema 2

Mais tarde, Cookson et alii ${ }^{6,7}$ desenvolveram outro procedimento mais eficiente para o preparo dos oxabiciclos $\mathbf{2 a}$ e $\mathbf{2 b}$ através da redução da $\alpha, \alpha^{\prime}$-dibromocetona $\mathbf{6}$ com NaI, na presença de furano (Esquema 3). Neste caso foi observada a formação de grande quantidade da furilcetona $2 \mathbf{c}$, resultante da reação de substituição eletrofílica entre o furano e o cátion oxialílico gerado in situ.

Para avaliar a generalidade desta cicloadição Cookson et $a l i i^{7}$ realizaram experimentos com ciclopentadieno e $\mathrm{N}$ metilpirrol cujos resultados (compostos 7a-b e 8) são mostrados no esquema 3 . No caso do $\mathrm{N}$-metilpirrol apenas o produto da substituição eletrofílica $\mathbf{8}$ foi isolado. 


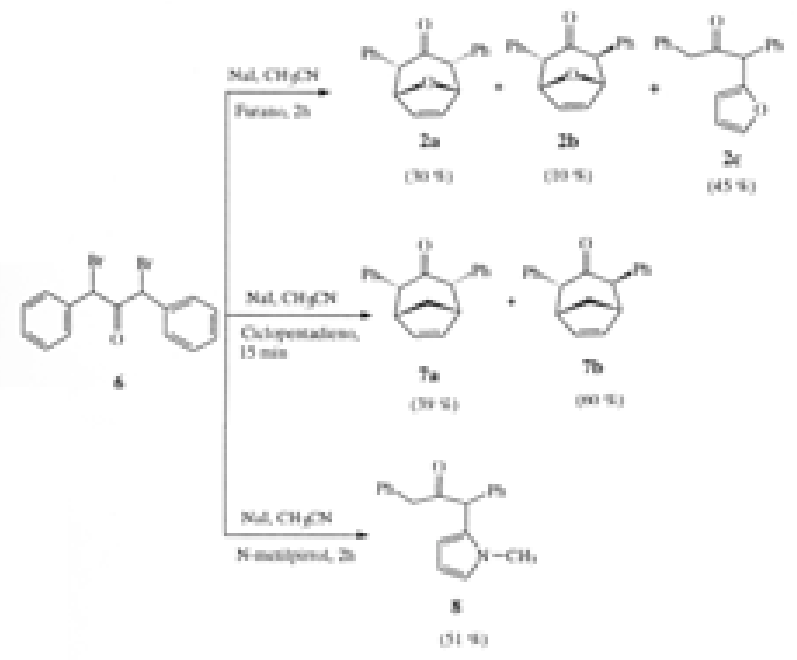

Esquema 3

A partir dos trabalhos pioneiros de Fort e Cookson demonstrando a viabilidade da construção de compostos carbocíclicos com sete membros, através da cicloadição [3+4], várias metodologias para gerar cátions oxialílicos foram descritas. $\mathrm{O}$ desenvolvimento destas metodologias devem-se principalmente aos trabalhos de Hoffmann ${ }^{2,9}$, Mann $^{8}$ e Noyori ${ }^{10,11}$.

A seguir serão apresentadas as principais técnicas descritas na literatura para o preparo de cátions oxialílicos e suas reações com dienos, mostrando, ilustrativamente, alguns exemplos da aplicação deste tipo de reação na síntese de moléculas complexas. Também alguns exemplos de reações de cicloadição [3+4] envolvendo cátions alílicos serão apresentados.

\section{FORMAÇ̃̃O E REATIVIDADE DE CÁTIONS OXIALÍLICOS}

Cátions oxialílicos são gerados in situ a partir de substratos como: $\alpha$-halocetonas, $\alpha, \alpha^{\prime}$-dialocetonas, $\alpha, \alpha, \alpha^{\prime}, \alpha^{\prime}$-tetraalocetonas e álcoois alílicos ativados, tratados com reagentes metálicos (ácidos de Lewis), os quais, na grande maioria das vezes, se complexam com o oxigênio da carbonila da cetona, estabilizando o enolato formado.

\subsection{Obtenção de cátions oxialílicos a partir de $\alpha$-halocetonas}

Nesta classe, os substratos mais comuns são $\alpha$-bromoalquil ou $\alpha$-bromoaril-cetonas, sendo que compostos clorados são também utilizados. Nesta categoria geral inclui-se o trabalho original de Fort ${ }^{3,5}$, o qual foi expandido por Mann et alii ${ }^{12,13}$. Essas reações são realizadas em solventes como etanol, 2,2,2trifluoroetanol e acetonitrila. O cátion oxialílico formado (Esquema 4) reage com furanos, levando, em geral, à formação de compostos bicíclicos ${ }^{14}$. Conforme mostrado na tabela 1 , outros reagentes podem ser utilizados para a geração de cátions oxialílicos a partir de $\alpha$-bromo ou $\alpha$-clorocetonas. O mecanismo da reação envolve a formação de um enolato, seguida da perda de um haleto (Esquema 4).

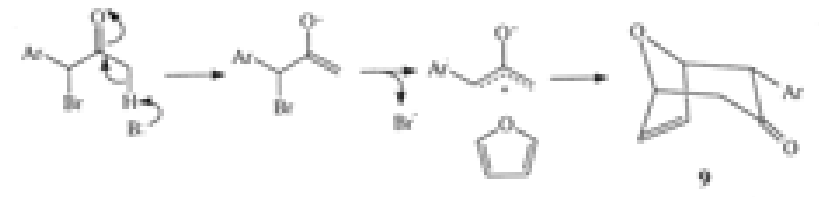

Esquema 4

\subsection{Obtenção de cátions oxialílicos a partir de $\alpha, \alpha^{\prime}$-dialocetonas e de $\alpha, \alpha, \alpha^{\prime}, \alpha^{\prime}$-tetraalocetonas}

A formação de cátions oxialílicos a partir de $\alpha, \alpha^{\prime}$-dialocetonas e $\alpha, \alpha, \alpha^{\prime}, \alpha^{\prime}$-tetraalocetonas pode ser realizada utilizando-se diferentes agentes redutores, conforme apresentado na tabela 2 .

$\mathrm{O}$ mecanismo geral dessas reações envolve, inicialmente, ataque do metal a um dos halogênios. O próprio metal, complexado com solvente (L) ou outros ligantes, interage com a carbonila e auxilia na retirada de um haleto, formando o cátion oxialílico correspondente.

No esquema 5 está mostrada, de maneira simplificada, a formação do cátion oxialílico 10, a partir de $\alpha, \alpha^{\prime}$-dialocetona, em presença de zinco.

Estudos teóricos ${ }^{33}$ sugerem que o cátion oxialílico 10 é instável na sua forma livre, isomerizando-se imediatamente para as formas 11 e $\mathbf{1 2}$ (isso também ocorreria nas reações com agentes redutores como $\mathrm{Fe}_{2}(\mathrm{CO})_{9}$ e $\left.\mathrm{Cu} / \mathrm{NaI}\right)$. A espécie 10 é mais estabilizada tanto pela presença de grupos elétron-doadores nas posições $\alpha$ e $\alpha^{\prime}$, quanto pelo aumento no caráter

Tabela 1. Reagentes utilizados para gerar cátions oxialílicos a partir de $\alpha$-halocetonas.

\begin{tabular}{|c|c|c|c|}
\hline Haleto & Condições Reacionais & Cátion gerado & Referência \\
\hline & $\begin{array}{l}\mathrm{Et}_{3} \mathrm{~N}, \mathrm{AgBF}_{4}, \\
\mathrm{CH}_{3} \mathrm{CN},-10^{\circ} \mathrm{C}\end{array}$ & & 12 \\
\hline & $\begin{array}{l}\mathrm{Et}_{3} \mathrm{~N}, \mathrm{CF}_{3} \mathrm{CH}_{2} \mathrm{OH}, \\
25^{\circ} \mathrm{C}\end{array}$ & & 13 \\
\hline$B_{2}$ & $\begin{array}{l}\mathrm{CF}_{3} \mathrm{CF}_{2} \mathrm{CH}_{2} \mathrm{ONa} \\
\mathrm{CF}_{3} \mathrm{CF}_{2} \mathrm{CH}_{2} \mathrm{OH}, \\
25^{\circ} \mathrm{C}\end{array}$ & & 15 \\
\hline a & $\begin{array}{l}\mathrm{Et}_{3} \mathrm{~N}, \mathrm{LiClO}_{4} \text {, éter } \\
25^{\circ} \mathrm{C}\end{array}$ & $k$ & 16,17 \\
\hline $\begin{array}{l}\mathrm{X}=\mathrm{H}, \mathrm{Cl} \\
\mathrm{R}=\text { alquil } \\
\mathrm{R}^{\prime}=\text { alquil }\end{array}$ & & & \\
\hline
\end{tabular}


Tabela 2. Reagentes utilizados para gerar cátions oxialílicos a partir de $\alpha, \alpha^{\prime}$ - dialocetonas e $\alpha, \alpha, \alpha^{\prime}, \alpha^{\prime}$-tetraalocetonas.

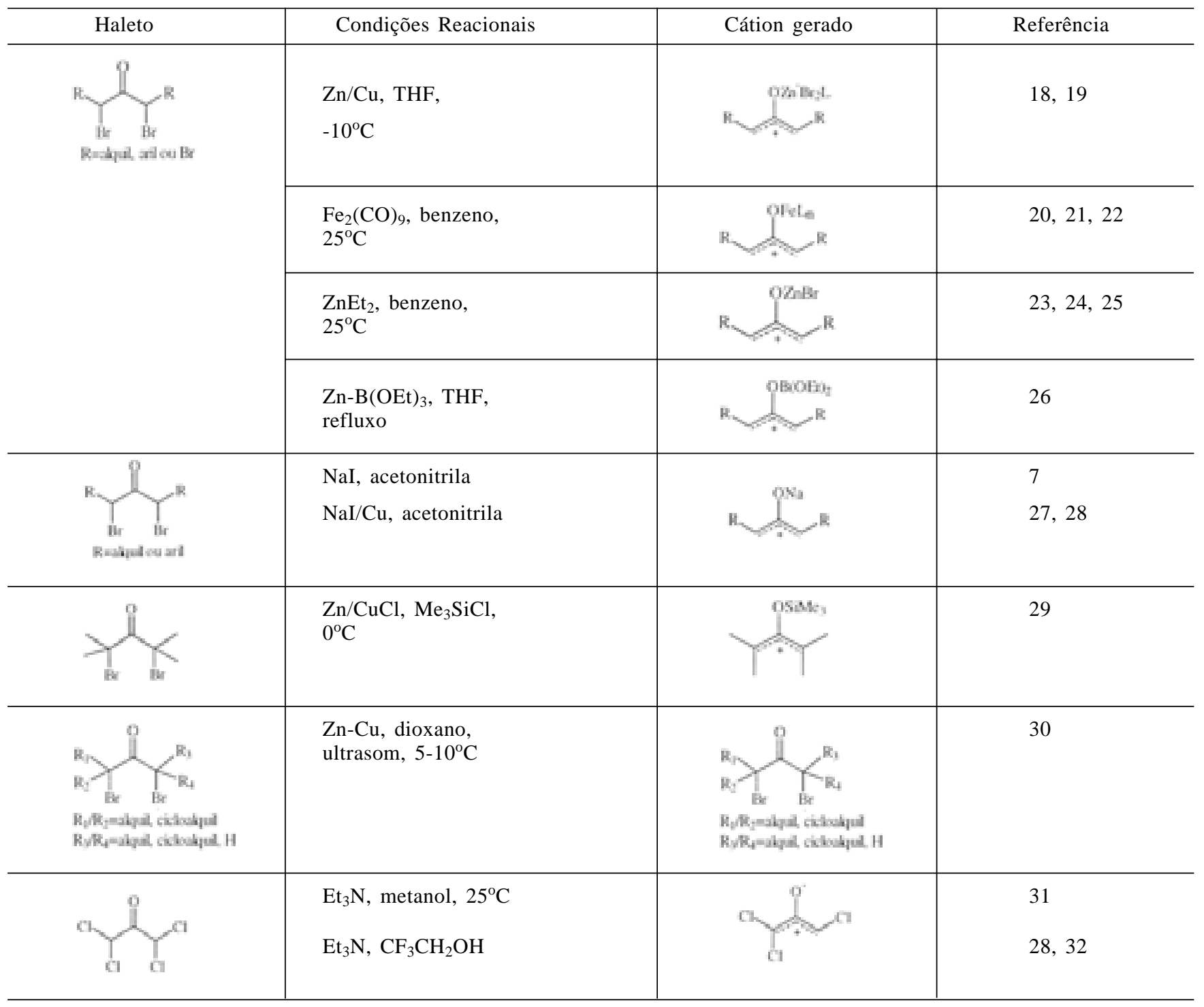

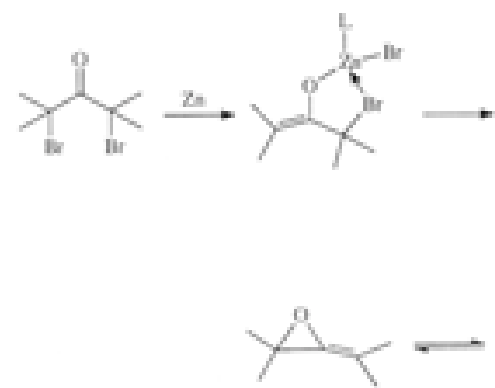

12

Esquema 5

covalente da ligação oxigênio-metal. Na maioria dos casos o metal pode ser $\mathrm{Zn}, \mathrm{Fe}, \mathrm{Na}, \mathrm{Li}$ ou $\mathrm{Si}^{8}$.

Hoffmann et alii ${ }^{18}$ estabeleceram os requerimentos estruturais mínimos necessários para a formação de cátions oxialílicos, estáveis o suficiente para participarem de reações de cicloadição $[3+4]$. Eles verificaram que dialocetonas como 13 não podem ser usadas, uma vez que elas levam à formação de cátions oxialílicos muito lábeis, que se polimerizam rapidamente. Por outro lado, compostos como 14-18, ou derivados destes, contendo mais grupos alquil, aril ou bromo nas posições $\alpha$ e/ou $\alpha$ ' são bons precursores de cátions oxialílicos, estáveis o suficiente para reagirem com dienos. Em resumo, quanto maior o número de grupos elétron-doadores nas posições $\alpha$ e $\alpha^{\prime}$, maior será a estabilidade do cátion resultante.
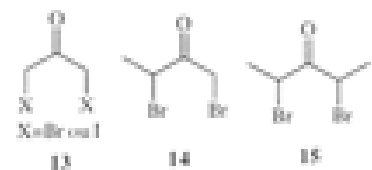

is

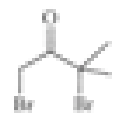

is
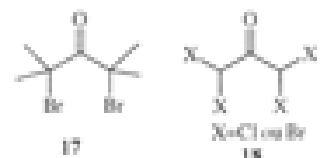

is

Outro método eficiente para gerar cátions oxialílicos, a partir de $\alpha, \alpha^{\prime}$-dibromocetonas, envolve o uso de NaI ou LiI, na presença de cobre, em acetonitrila ${ }^{27}, 33-35$. A reação se inicia pelo deslocamento $\left(A_{N} D_{N}\right)^{9,36}$ do brometo pelo iodeto. O processo é rápido e a precipitação de $\mathrm{NaBr}$ é observada quando se utiliza acetonitrila como solvente. A próxima etapa envolve a remoção redutiva de um átomo de iodo pelo iodeto, formando iodo molecular, o qual é capturado pelo cobre ou, algumas vezes, pelo mercúrio. Subsequentemente ao processo discutido, ocorre a eliminação $\left(\mathrm{D}_{\mathrm{N}}\right)$ de um íon iodeto, resultando na formação do cátion oxialílico 19 (Esquema 6$)^{9,37}$.

Se no caso de formação do cátion oxialílico 19, o metal utilizado for o lítio, a espécie se torna mais eletrofílica, e em 


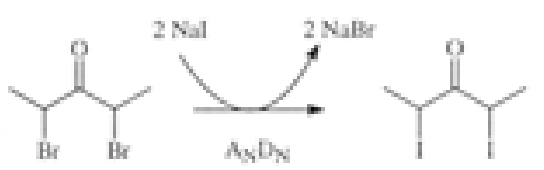

KC
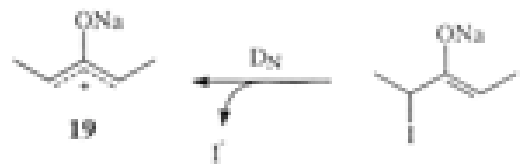

Esquema 6

reações com dienos ricos em elétrons como o N-metilpirrol, observa-se apenas a formação de produtos resultantes de substituição eletrofílica (cf. rota BC no esquema 17) (1,9 $^{8}$.

A utilização de $\mathrm{Fe}_{2}(\mathrm{CO})_{9}$ para gerar cátions oxialílicos a partir de $\alpha, \alpha^{\prime}$-dibromocetonas e $\alpha, \alpha, \alpha^{\prime}, \alpha^{\prime}$-tetrabromocetonas foi desenvolvida por Noyori et alii ${ }^{19,20,38-40}$. Este reagente pode ser utilizado com uma grande variedade de polibromocetonas, e dienos cíclicos (Esquema 7) e acíclicos.

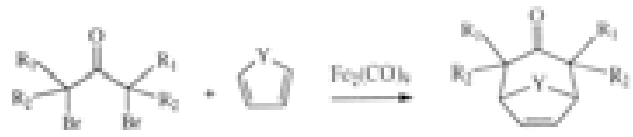

$\mathrm{R}_{1}=$ alquil, $\mathrm{R}_{2}=$ alquil, aril, $\mathrm{H}$ ou $\mathrm{Br}$ $\mathrm{Y}=\mathrm{CH}_{2}, \mathrm{O}, \mathrm{NCOCH}_{3}$, etc.

\section{Esquema 7}

Como o complexo $\mathrm{Fe}_{2}(\mathrm{CO})_{9}$ é essencialmente neutro, diferentes grupos funcionais como carbonilas (aldeídos e cetonas), éster, amida, ciano, éter, etc. podem estar presentes tanto na cetona como no dieno.

Vários estudos sobre o mecanismo da reação entre o $\mathrm{Fe}_{2}(\mathrm{CO})_{9}$ e polibromocetonas foram realizados e a formação dos intermediários $\mathbf{2 0}$ e $\mathbf{2 1}$ foi comprovada por Noyori ${ }^{10}$ e Noyori et alii $^{20}$ (Esquema 8).
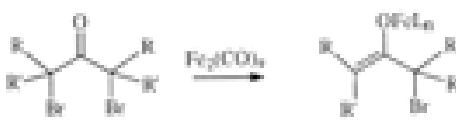

20

1. aBi, $\mathrm{CO}$ solvente, ets:

\section{Esquema 8}

Recentemente, Mann e Barbosa ${ }^{23}$ desenvolveram um novo método de geração de cátions oxialílicos a partir de $\alpha, \alpha^{\prime}$ dibromocetonas e $\alpha, \alpha, \alpha^{\prime}, \alpha^{\prime}$-tetrabromocetonas, por tratamento com dietilzinco. Os cátions gerados por este método reagem com furano, alquilfurano e N-carboximetilpirrol, resultando na formação dos biciclos correspondentes.

Este método ${ }^{24}$ permite o preparo de biciclos do tipo 22 e 23, a partir de 1,1,3,3-tetrabromo-4-metilpentan-3-ona e 1,1,3,3-tetrabromoacetona, na escala de 50-100 mmol, com rendimentos da ordem de 50-60\% (Esquemas 9 e 10). O único método até então disponível para o preparo desses compostos envolvia o uso de $\mathrm{Fe}_{2}(\mathrm{CO})_{9}$, que é muito caro e tóxico ${ }^{11}$.

$\mathrm{O}$ uso de $\mathrm{Et}_{2} \mathrm{Zn}$ permite também preparar cicloadutos como (24-27) (Esquema 11) e 28-29 (Esquema 12) diretamente a partir de furilálcool, sem a necessidade de proteção da hidroxila $^{25}$, diferentemente de todos os métodos até então conhecidos. Deve ser notado que quando os cátions oxialílicos
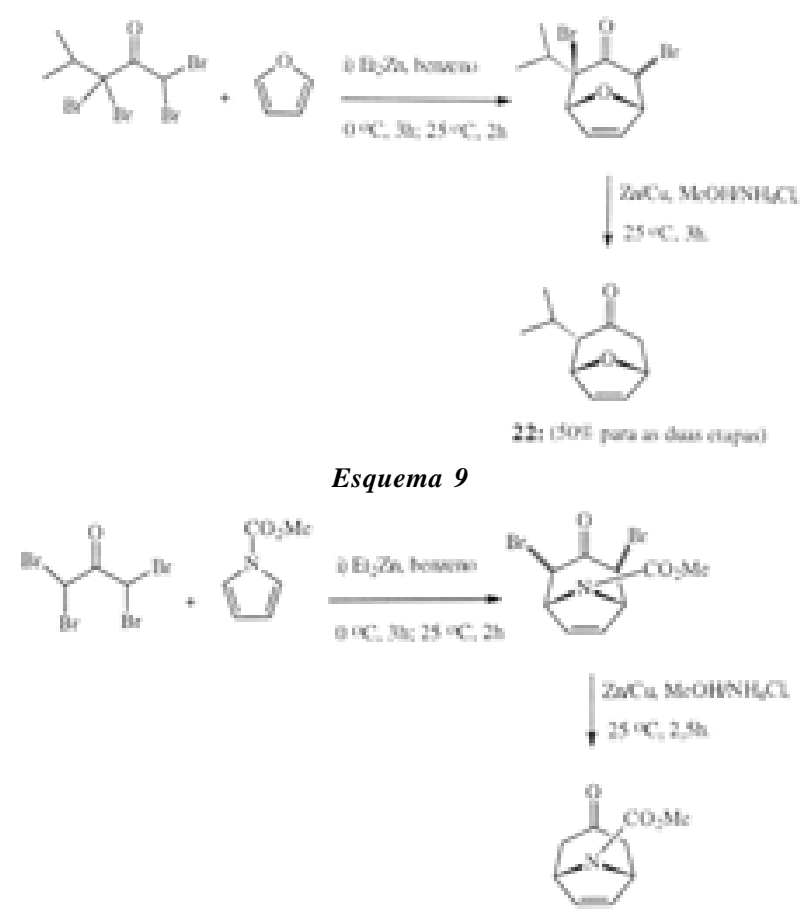

23: (5s,3) pura a duas chapus)

Esquema 10

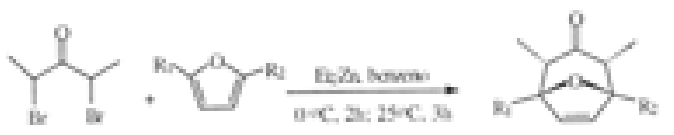

24: $\mathrm{R}_{1}=\mathrm{R}_{2}=\mathrm{CH}_{2} \mathrm{OH}(11 \%)$

25: $\mathrm{R}_{1}=\mathrm{H}, \mathrm{R}_{2}=\mathrm{CH}_{2} \mathrm{CH}_{2} \mathrm{CH}_{2} \mathrm{OH}(90 \%)$

26: $\mathrm{R}_{1}=\mathrm{H}, \mathrm{R}_{2}=\mathrm{CH}_{2} \mathrm{CH}_{2} \mathrm{CH}_{2} \mathrm{OAc}(85 \%)$

27: $\mathrm{R}_{1}=\mathrm{H}, \mathrm{R}_{2}=\mathrm{CH}=\mathrm{CHCH}_{2} \mathrm{OH}(66 \%)$

Esquema 11

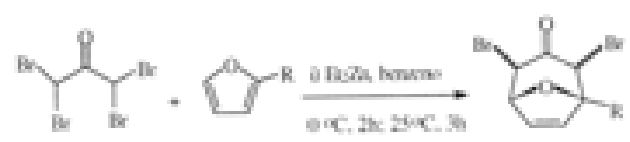

Zucie shownisa

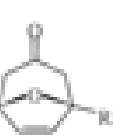

28: $\mathrm{R}=\mathrm{CH}_{2} \mathrm{CH}_{2} \mathrm{CH}_{2} \mathrm{OAc}(51 \%)$

29: $\mathrm{R}=\mathrm{CH}=\mathrm{CHCH}_{2} \mathrm{OH}(55 \%)$

Esquema 12

são gerados a partir de 1,1,3,3-tetrabromocetona (Cf. Esquema 9 e10), os oxabiciclos formados são dibromados. O tratamento dos mesmos com $\mathrm{Zn} / \mathrm{Cu}$ na presença de $\mathrm{MeOH} / \mathrm{NH}_{4} \mathrm{Cl}$ resulta na obtenção dos produtos reduzidos. $\mathrm{O}$ mecanismo proposto ${ }^{23}$ para a geração de cátions oxialílicos, a partir de polibromocetonas e dietilzinco é apresentado no esquema 13.

\subsection{Obtenção de cátions oxialílicos ou alílicos a partir de outros substratos}

Vários substratos, além de polialocetonas, podem ser utilizados para gerar cátions oxialílicos ou mesmo cátions alílicos, conforme mostrado na tabela 3 . 


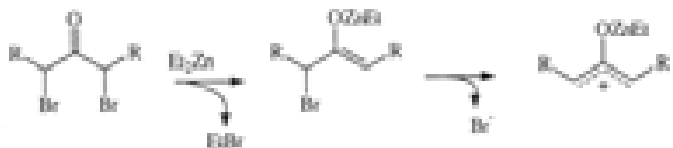

Esquema 13

Um dos primeiros métodos para cicloadição entre dienos conjugados e cátions alílicos foi desenvolvido por Hoffmann et alii $^{41}$, usando iodetos alílicos 30, na presença de sais de prata, o que favorece a formação do intermediário 31, produzindo os compostos $\mathbf{3 2}$ e $\mathbf{3 3}$ através da perda de uma molécula de HX (Esquema 14).

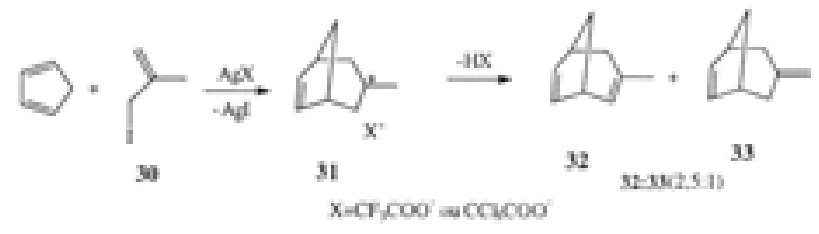

Esquema 14
A reação de iodetos alílicos com sais de prata $\left(\mathrm{AgOCOCF}_{3}\right.$ ou $\mathrm{AgOCOCCl} l_{3}$ ) proporciona a precipitação do sal insolúvel AgI, tornando irreversível a formação do cátion. O tricloroacetato e trifluoroacetato, nestas reações, interagem com o cátion alílico formado, estabilizando-o pela formação de um par de íons separados pelo solvente ${ }^{41}$ :

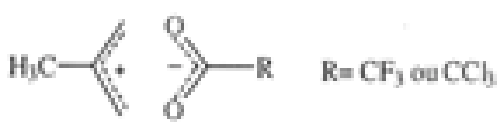

Brometos e cloretos alílicos podem também ser utilizados para essa reação, todavia os iodetos são mais reativos ${ }^{2}$. Outros sais de prata como $\mathrm{AgClO}_{4}{ }^{42,43}$ e $\mathrm{AgBF}_{4}{ }^{52}$ podem também ser utilizados (Tabela 3).

Álcoois alílicos podem ser ativados pela formação do trifluoroacetato correspondente. O trifluoroacetato sofre uma reação do tipo $\mathrm{D}_{\mathrm{N}}$ seguindo-se a cicloadição do intermediário com dienos ${ }^{46}$. O cloreto de titânio (IV) também tem sido usado efetivamente para gerar cátion oxialílico, a partir de álcoois alílicos e de derivados $p$-toluenossulfonatos ${ }^{17,29,46-51}$.

Tabela 3. Reagentes utilizados para gerar cátions oxialílicos e alílicos a partir de substratos variados.

\begin{tabular}{|c|c|c|c|}
\hline Substrato & Condições Reacionais & Cátion gerado & Referência \\
\hline Ornes & $\mathrm{AgClO}_{4}$, éter, $0^{\circ} \mathrm{C}$ & OTMS & 42,43 \\
\hline OTMS & $\begin{array}{l}\mathrm{TiCl}_{4} \text { ou } \\
\mathrm{SnCl}_{4} \text { ou } \\
\mathrm{TMSOTf} \text { ou } \\
\mathrm{BF}_{3} \cdot \mathrm{Et}_{2} \mathrm{O}\end{array}$ & $\begin{array}{l}\mathrm{R}=\mathrm{OTiCl}_{4}^{-} \text {ou } \\
\mathrm{R}=\mathrm{OSnCl}_{4}^{-} \text {ou } \\
\mathrm{R}=\mathrm{OTMSOTf}^{-} \text {ou } \\
\mathrm{R}=\mathrm{OBf}_{3}^{-}\end{array}$ & 44 \\
\hline & $\begin{array}{l}\mathrm{AgOCOCF}_{3} \\
\text { benzeno, } \\
-78^{\circ} \mathrm{C}\end{array}$ & & 41 \\
\hline OE & $\begin{array}{l}\left(\mathrm{CF}_{3} \mathrm{CO}\right)_{2} \mathrm{O} \\
\mathrm{Pr}_{2} \mathrm{EtN}\end{array}$ & & 45 \\
\hline & $\begin{array}{l}\mathrm{TiCl}_{4} \\
\mathrm{CH}_{2} \mathrm{Cl}_{2} \\
-78^{\circ} \mathrm{C}\end{array}$ & & $\begin{array}{l}46,47,48, \\
49,50\end{array}$ \\
\hline & $\mathrm{AlMe}_{3}$ ou hv & & 51 \\
\hline Mes & $\begin{array}{l}\mathrm{TiCl}_{4}, \\
\mathrm{PhNHMe} \\
\mathrm{CH}_{2} \mathrm{Cl}_{2},-20^{\circ} \mathrm{C}\end{array}$ & & 29,45 \\
\hline & $\begin{array}{l}\mathrm{AgBF}_{4} \\
\mathrm{CH}_{2} \mathrm{Cl}_{2} \\
-78^{\circ} \mathrm{C}\end{array}$ & & 52 \\
\hline
\end{tabular}


Apesar de todos os exemplos apresentados até o momento envolverem apenas processos intermoleculares, várias cicloadições intramoleculares [3+4] já foram descritas ${ }^{17,46-51}$.

Harmata et alii $^{48,53}$ mostraram que a reação de alcoxialilsulfonas do tipo 34 e $36 \mathrm{com} \mathrm{TiCl}_{4}$, ou outros ácidos de Lewis, resultam na formação de estruturas cíclicas tipo 35 e 37/38, com bons rendimentos (Esquema 15).

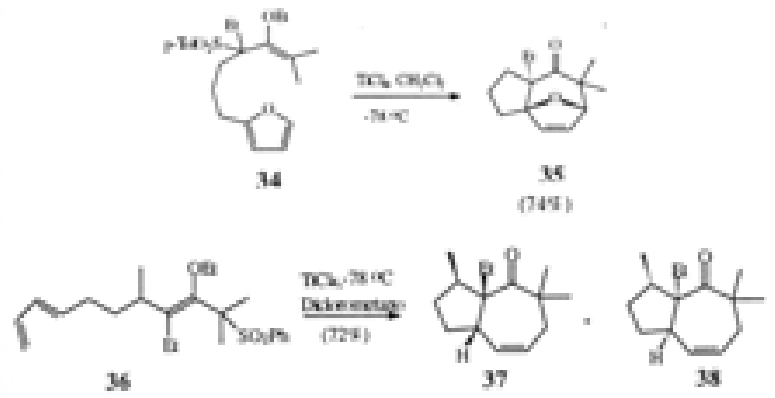

Esquema 15

Outro exemplo de cicloadição intramolecular [3+4], propiciando a obtenção dos compostos 39 e 40, foi descrito por Hoffmann et alii $^{54}$ (Esauema 16).

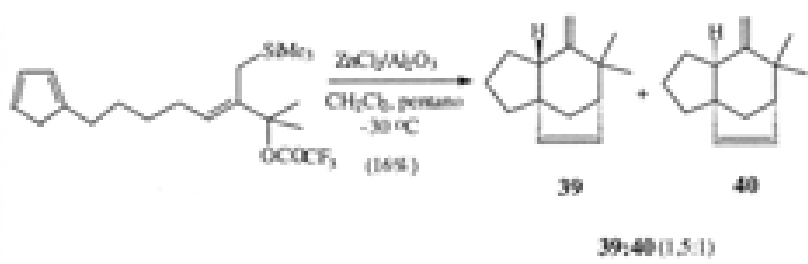

Esquema 16

\subsection{Mecanismos de cicloadição de cátions oxialílicos com 1,3-dienos}

Nos itens anteriores foram apresentados diversos métodos utilizados para gerar cátions oxialílicos (e alílicos). Uma vez formado, o cátion pode reagir com 1,3-dienos de diferentes maneiras, conforme representado no esquema 17 , levando à produção dos seguintes tipos de compostos ${ }^{9,37}$ :

i) Cicloadutos de sete membros (42) (rotas $\mathrm{AA}^{\prime}$ e BB');

ii) Produtos derivados de substituição eletrofílica (45) sobre o dieno, (rota BC);

iii) Compostos derivados da captura nucleofílica intermolecular $(46,47)$ (rota BE) e intramolecular 48 e 49 (rota BD).

A rota $\mathrm{AA}^{\prime}$, corresponde a uma cicloadição [3+4], que resulta na formação do cicloaduto $\mathbf{4 3}$, através dos estados de transição denominados de compacto (tipo barco) ou extendido (tipo cadeira) (Esquema 18).

De acordo com Hoffmann ${ }^{2,18}$, os cátions oxialílicos podem se apresentar nas conformações em $\mathrm{W}, \mathrm{U}$ ou foice, sendo que a conformação em W é a mais estável e portanto a predominante.

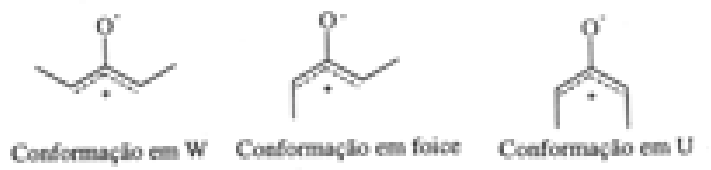

Considerando portanto a reação entre o cátion oxialílico dissubstituído na conformação em $\mathrm{W}$ e um dieno cíclico, o cicloaduto resultante poderá ter estereoquímica $\alpha \alpha(\mathbf{5 0})$ ou $\beta \beta$

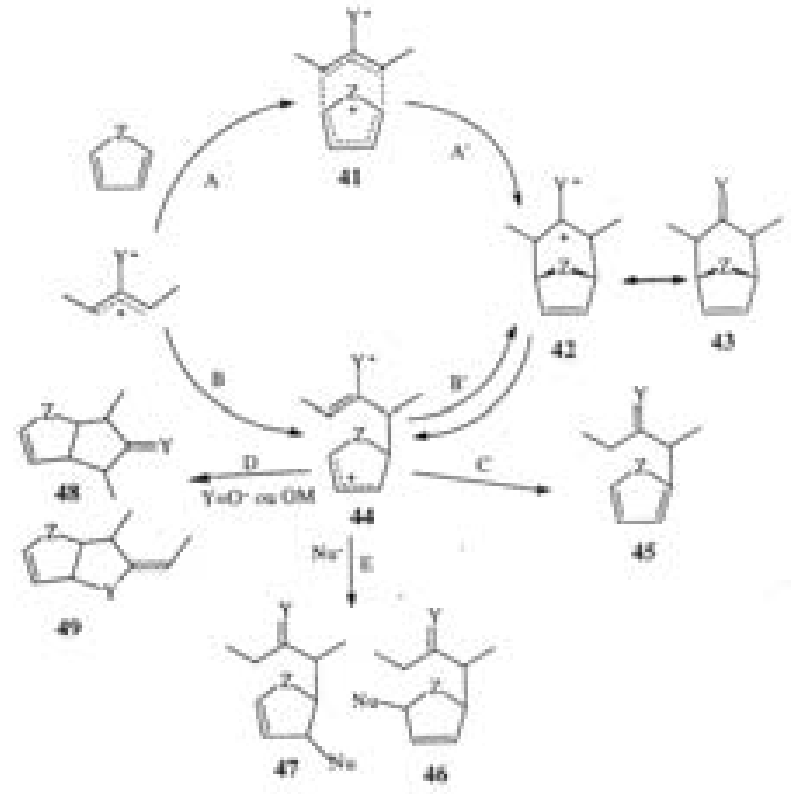

$\mathrm{Y}=\mathrm{O}-\mathrm{Metal}, \mathrm{OsiMe}_{3}, \mathrm{OB}(\mathrm{OEt})_{2}$

$\mathrm{Z}=\mathrm{CH}_{2}, \mathrm{O}, \mathrm{N}-\mathrm{Me}, \mathrm{N}-\mathrm{COMe}$

Esquema 17

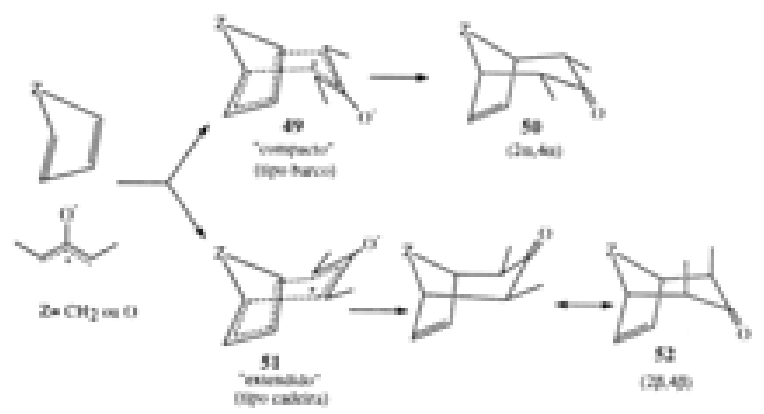

Esquema 18

(52) (Esquema 18). A razão entre as quantidades formadas dos estereoisômeros $\alpha \alpha$ e $\beta \beta$ é um reflexo da contribuição de cada estado de transição (compacto ou extendido) para o mecanismo da cicloadição.

Analisando os resultados da reação entre ciclopenta-1,3dieno com 2,4-dibromopentan-3-ona com diferentes agentes redutores (Esquema 19) Hoffmann ${ }^{9}$ concluiu que, em geral, cátions mais eletrofílicos têm maior tendência a reagir pelo estado de transição extendido. A eletrofilicidade do cátion depende da natureza da espécie $\mathrm{M}\left(\mathrm{M}=\mathrm{Fe}, \mathrm{Zn}, \mathrm{Li}, \mathrm{SiMe}_{3}\right.$, $\mathrm{B}(\mathrm{OEt})_{2}$, etc.) ligada ao oxigênio. Quanto maior for o caráter covalente da ligação O-M, mais eletrofílico será o cátion.

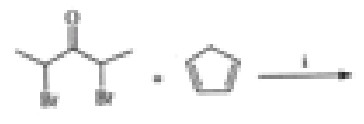

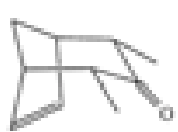

$530=$

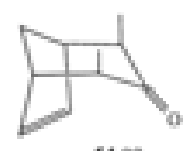

54 ab
i) $\mathrm{NaI} / \mathrm{Cu}, \mathrm{CH}_{3} \mathrm{CN}$
$\alpha \alpha: \beta \beta(6,4: 1)$
i) $\mathrm{Zn} / \mathrm{Cu}, \mathrm{DME}$
$\alpha \alpha: \beta \beta(1,7: 1)$
i) $\mathrm{Fe}_{2}(\mathrm{CO})_{9}, \mathrm{C}_{6} \mathrm{H}_{6}$ $\alpha \alpha: \beta \beta(0,89: 1)$

Esquema 19

Já para a reação entre furano e 2,4-dibromopentan-3-ona, 
com três agentes redutores, foi observada a formação de três cicloadutos isoméricos $\alpha \alpha, \beta \beta$ e $\alpha \beta^{2,9}$ (Esquema 20).
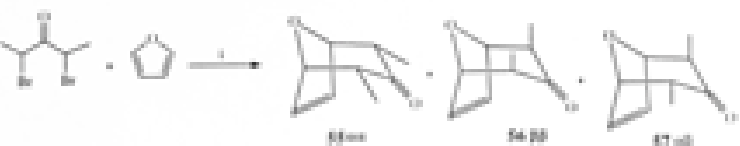

i) $\mathrm{NaI} / \mathrm{Cu}, \mathrm{CH}_{3} \mathrm{CN}$

$\alpha \alpha: \beta \beta: \alpha \beta(90,9: 3,0: 6,1)$

i) $\mathrm{Zn} / \mathrm{Cu}, \mathrm{DME}$ $\alpha \alpha: \beta \beta: \alpha \beta(73,9: 8,7: 17,9)$

i) $\mathrm{Fe}_{2}(\mathrm{CO})_{9}, \mathrm{C}_{6} \mathrm{H}_{6}$

$\alpha \alpha: \beta \beta: \alpha \beta(44,9: 0,0: 56,0)$

Esquema 20

Para explicar a formação do isômero $\alpha \beta$, Hoffmann ${ }^{9}$ sugeriu que o cátion oxialílico é inicialmente formado na conformação $\mathrm{W}$, mas o mesmo perde parcialmente essa conformação quando o intermediário 44 (Esquema 17) é formado. $\mathrm{O}$ ataque pelo enolato a qualquer uma das faces do sistema cíclico é possível e resulta na formação dos três adutos isoméricos. Neste caso Hoffmann concluiu que a reação se processa, em etapas, pelo mecanismo BB'. O intermediário $\mathbf{4 4}$ pode sofrer outras transformações e ser convertido nos compactos do tipo 45, 46, 47, 48 e 49. Todavia essas reações não são de interesse para a presente revisão.

\section{APLICAÇÕES SINTÉTICAS}

Conforme apresentado nas seções anteriores, uma grande variedade de reagentes é disponível para gerar cátions oxialílicos. A escolha adequada dos precursores dos cátions oxialílicos (58) e dos dienos (59), permite síntese de cicloeptenos 60 funcionalizados em qualquer posicão (Esquema 21).
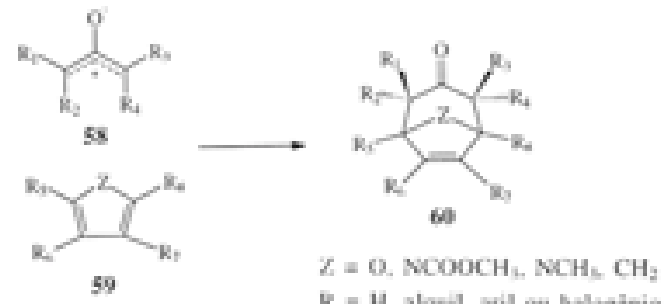

$\mathrm{K}=1 \mathrm{H}$, alg̣ul, arill ou halogęnio

Esquema 21

A rigidez da estrutura bicíclica $\mathbf{6 0}$ pode ser utilizada para controlar a estereoquímica em transformações posteriores. No caso da cicloadição envolvendo dienos acíclicos, essa possibilidade não existe, uma vez que o cicloaduto formado é monocíclico e, portanto, bastante mais flexível ${ }^{9}$.

Outra vantagem dessas reações de cicloadição [3+4] é que geram cicloadutos para os quais há grande potencial de transformações químicas ${ }^{8}$. Por exemplo, mesmo o mais simples dos biciclos (61) obtido por essa metodologia, pode sofrer uma gama enorme de transformações químicas, conforme ilustrado na figura 1. Nos exemplos que se seguem, várias dessas transformações são apresentadas, ilustrando a gama de aplicações em síntese das mais diferentes substâncias.

$\mathrm{Na}$ figura 2 são mostrados diversos produtos obtidos por Barbosa et $^{\text {aliit }}{ }^{23,24,55-61}$ a partir do biciclo com a estrutura genérica $\mathbf{6 0}$.

\subsection{Obtenção de compostos bifenílicos e de troponas}

Devido à atividade antitumoral apresentada pela colchicina $^{62}(62)$ e seu análogo 63, Mann et alii $^{13}$ tentaram preparar ariltroponas por meio da clivagem da ligação éter de vários 2-arilbiciclos (64). Entretanto, em vez de ariltroponas, eles obtiveram 1-aril-3-furilpropan-2-onas (65), com bom rendimento (Esquema 22).

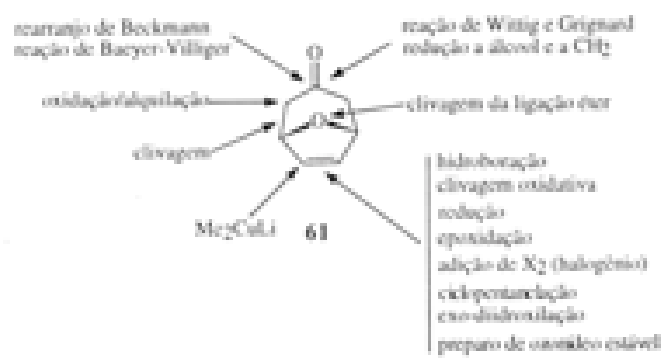

Figura 1. Reações possíveis de serem realizadas com o biciclo $6 \mathbf{1}^{37}$.

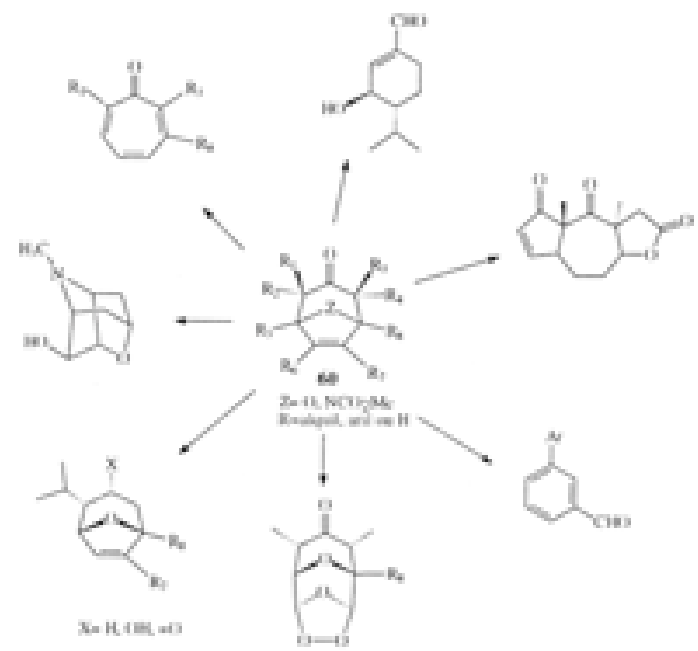

Figura 2. Transformações realizadas por Barbosa et alii ${ }^{24,55-61}$ a partir do biciclo com estrutura genérica $\mathbf{6 0}$
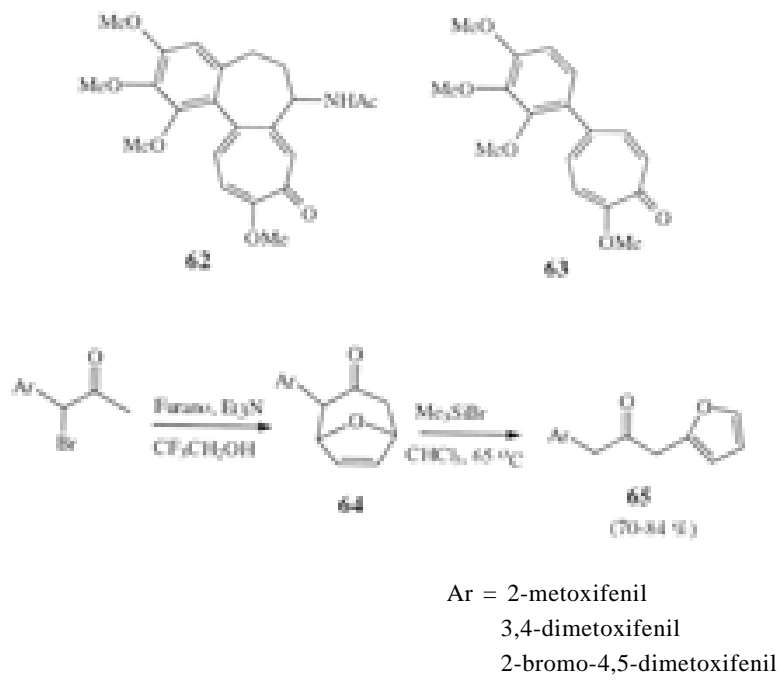

Esquema 22

Em uma outra tentativa de se preparar ariltroponas, partindo do 8-oxabicicl, 3.2.1] oct-6-en-3-ona ( 66), Mann et alii ${ }^{13}$ conseguiram apenas obter as bifenilas $68 \mathbf{a}$ e $\mathbf{6 8 b}$, utilizando estratégia mostrada no esquema 23 . O mecanismo proposto para o rearranjo da cetona 69 em bifenilas do tipo 68 é apresentado no esquema 24.

Outros rearranjos dos oxabicíclicos obtidos por cicloadição $[3+4]$ foram registrados na literatura ${ }^{63,64}$. Assim, essa metodologia permite o preparo fácil de diversas biarilas polissubstituídas.

Em um trabalho subsequente, Barbosa et alii ${ }^{55,60}$ descreveram um método para a síntese de troponas (71a-e) a partir de oxabiciclos substituídos 70a-e (Esquema 25). Os oxabiciclos 


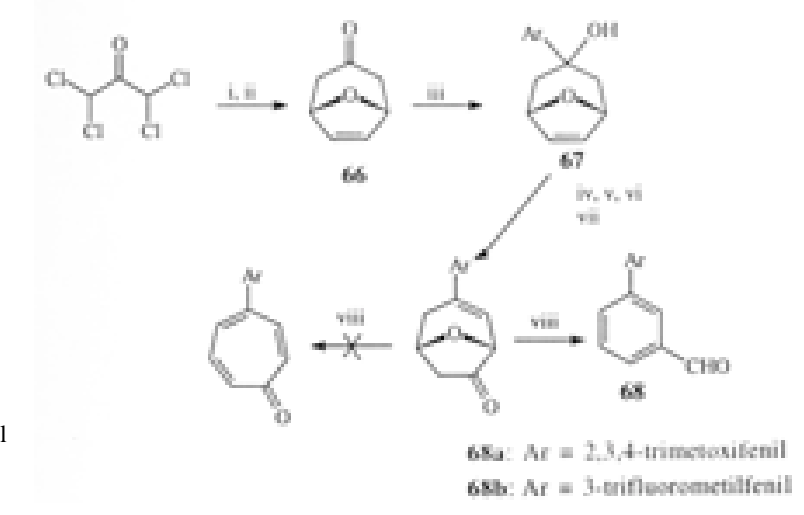

i) $\mathrm{Et}_{3} \mathrm{~N}, \mathrm{CF}_{3} \mathrm{CH}_{2} \mathrm{OH}$, furano; ii) $\mathrm{ArLi}$ ou $\mathrm{ArMgBr}$; iv) $\mathrm{BF}_{3}$; v) $\mathrm{H}_{2} \mathrm{O}_{2}, \mathrm{NaOH}$; vi) $\mathrm{H}+$; vii) $\mathrm{CrO}_{3} /$ piridina; viii) $\mathrm{HBr}$ (aq. 48\%), 68a, 85\%;68b,64\%

Esquema 23

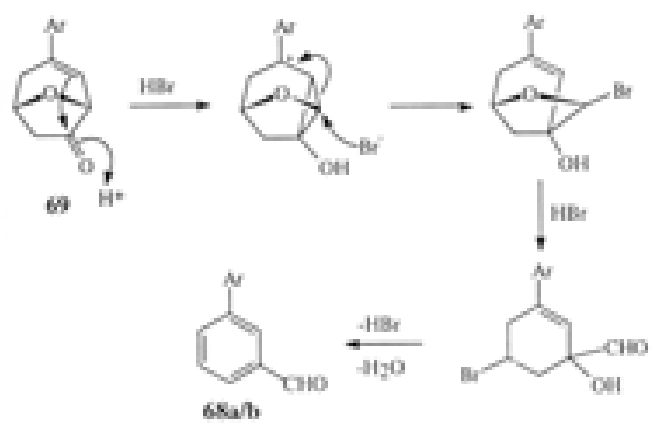

Esquema 24

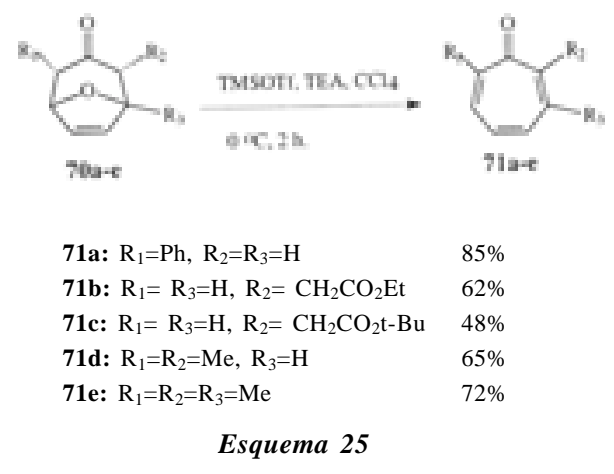

(70a-e) foram preparados utilizando-se diversos métodos de cicloadição já discutidos ${ }^{8,9}$. Essa metodologia pode vir a ser utilizada para a obtenção de análogos da colchicina (62).

Outro método para a obtenção de troponas alquiladas, desenvolvido por Takaya et alii ${ }^{65}$, envolve o tratamento de cicloeptenonas com hidrotribrometo de pirrolidona, seguido de aquecimento com LiCl/DMF. Nestes casos as cicloeptenonas são obtidas pela cicloadição entre dienos acíclicos e polibromocetonas, na presença de $\mathrm{Fe}_{2}(\mathrm{CO})_{9}$. $\mathrm{O}$ dieno pode também ser utilizado na forma de um complexo com $\mathrm{Fe}(\mathrm{CO})_{3}$. Um exemplo ilustrativo dessa metodologia é apresentado no esquema $26^{65}$.

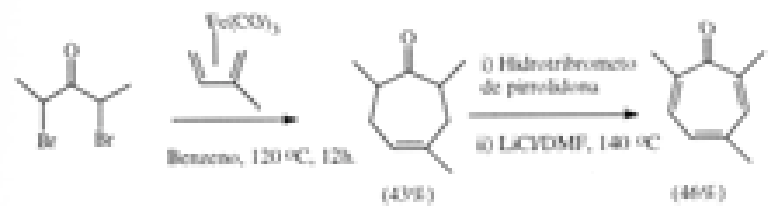

Esquema 26

\subsection{Obtenção de ozonídeos}

Oxabiciclos foram também utilizados para obtenção de análogos da artemisinina (72), um composto ativo contra malária $^{66-68}$. O esquema 27 apresenta a síntese de uma série de ozonídeos estáveis 73, que foram obtidos recentemente. Estes ozonídeos foram testados para avaliação da atividade antimalárica, utilizando-se uma linhagem de Plasmodium falciparum resistente à cloroquina. A tabela 4 apresenta os resultados dos testes biológicos ${ }^{57,61}$.
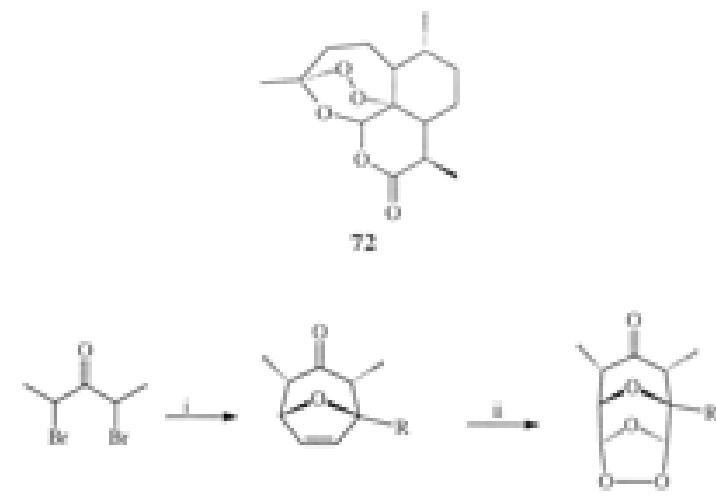

73

i) $\mathrm{Cu}, \mathrm{NaI}$, alquilfurano, acetonitrila $\left(60-80 \%\right.$ para $\mathrm{R}=\mathrm{H}, \mathrm{Et}, \mathrm{C}_{7} \mathrm{H}_{15} ; \mathrm{CH}_{2} \mathrm{OAc}$, $\mathrm{CH}\left(\mathrm{OMe}\right.$ ); Et $2 \mathrm{Zn}$, benzeno (52\% para $\mathrm{R}=\mathrm{CH}_{2} \mathrm{CH}_{2} \mathrm{CH}_{2} \mathrm{OAc}, \mathrm{CH}_{2} \mathrm{CH}_{2} \mathrm{CH}_{2} \mathrm{Vl}$ ); ii) $\mathrm{O}_{3}, 0^{\circ} \mathrm{C}$, diclorometano, $100 \%$.

\section{Esquema 27}

Tabela 4. Atividade antimalárica de alguns ozonídeos ${ }^{57,61}$.

\begin{tabular}{ll}
\hline Substância & $\mathrm{IC}_{50}\left(\mu \mathrm{g} . \mathrm{cm}^{-3}\right)$ \\
\hline 72: artemisinina & $10^{-3}$ \\
73: $\mathrm{R}=\mathrm{H}$ & 18 \\
73: $\mathrm{R}=\mathrm{Et}$ & 12 \\
73: $\mathrm{R}=\mathrm{C}_{7} \mathrm{H}_{15}$ & 26 \\
73: $\mathrm{R}=\mathrm{CH}_{2} \mathrm{OAc}$ & inativo $(>500)$ \\
73: $\mathrm{R}=\mathrm{CH}_{(\mathrm{OMe}) \mathrm{Ph}}$ & inativo $(>500)$ \\
73: $\mathrm{R}=\mathrm{CH}_{2} \mathrm{CH}_{2} \mathrm{CH}_{2} \mathrm{OAc}$ & 3 \\
\hline
\end{tabular}

\subsection{Obtenção de alcalóides tropânicos}

O estudo de sínteses de alcalóides tropânicos tem também recebido grande atenção por causa de seus usos terapêuticos ${ }^{69}$. Um dos métodos de síntese destes alcalóides envolve a cicloadição entre $\mathrm{N}$-carbometoxipirrol $(\mathbf{7 4})^{10,23,69-71}$ e o cátion oxialílico produzido a partir da reação entre 1,1,3,3-tetrabromopropanona e $\mathrm{Fe}_{2}(\mathrm{CO})_{9}$.

$\mathrm{O}$ esquema 28 mostra a síntese da 6,7-deidrotropina (76) e sua transformação em diversas outras substâncias: hiosciamina (77), escopina (78) e teloidina (79).

Uma rota de síntese mais simples, propiciada pelo uso de dietilzinco, um reagente menos tóxico do que $\mathrm{Fe}_{2}(\mathrm{CO})_{9}$, foi usada na síntese da escopolina (80) (Esquema 29) ${ }^{23}$.

\subsection{Estudos visando a obtenção de lactonas sesquiterpênicas}

O interesse na síntese de lactonas sesquiterpênicas, do tipo pseudoguaianolídeo e análogos, deve-se à descoberta de que muitos compostos desta classe possuem atividade citotóxica e anti-tumoral $^{72-84}$. Dentre estes, a helenalina $(\mathbf{8 1})$ é a que apresenta maior atividade anti-tumoral.

Visando obter compostos estruturalmente parecidos com a helenalina, para fins de avaliação da atividade antitumoral, Barbosa 

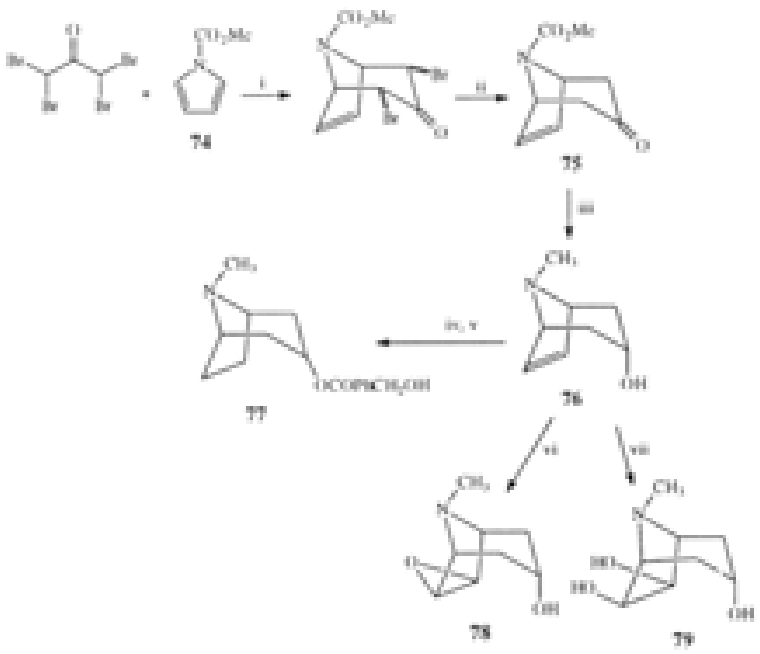

i) $\mathrm{Fe}_{2}(\mathrm{CO})_{9}$, benzeno, $70 \%$; ii) $\mathrm{Zn} / \mathrm{Cu}, \mathrm{MeOH} / \mathrm{NH}_{4} \mathrm{Cl}, 100 \%$; iii) DIBAH, $87 \%$; iv) $\mathrm{CIOCCHPhCH}_{2} \mathrm{OH}, 88 \%$; v) $\mathrm{H}_{2} / \mathrm{Pd} / \mathrm{C}, 99 \%$; vi) $\mathrm{CF}_{3} \mathrm{CO}_{3} \mathrm{H}$; vii) $\mathrm{KMnO}_{4}$

Esquema 28

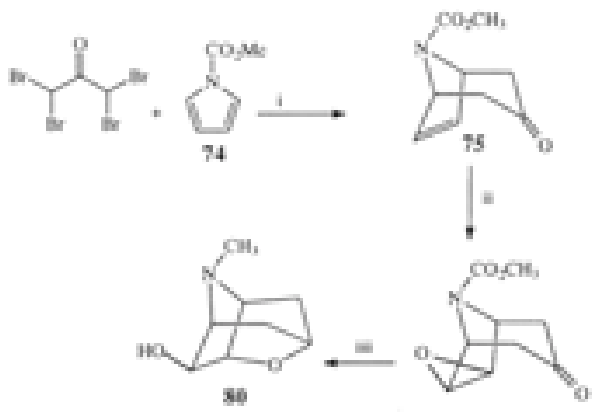

i) $\mathrm{Et}_{2} \mathrm{Zn}$, benzeno, $\mathrm{Zn} / \mathrm{Cu}, \mathrm{MeOH} / \mathrm{NH}_{4} \mathrm{Cl}, 50 \%$; ii) $\mathrm{mCPBA}, 83 \%$; iii) DIBAH, $53 \%$

Esquema 29

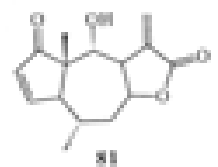

e $\mathrm{Mann}^{58}$ desenvolveram uma rota sintética que resultou no preparo da lactona (83), utilizando o 2,4-dimetil-8-oxabiciclo [3.2.1] oct-6-en-3-ona (82) como material de partida, conforme mostrado no esquema 30. Essa metodologia poderá ser explorada para o preparo de lactonas sesquiterpênicas naturais e análogos.

\subsection{Estudos visando a obtenção de análogos oxigenados do ácido helmintospórico}

Os sesquiterpenos helmintosporol $(\mathbf{8 4})^{85}$, helmintosporal $(\mathbf{8 5})^{86}$ e ácido helmintospórico (86), isolados de Helmintosporium sativum, apresentam efeito sobre o desenvolvimento de diversas plantas ${ }^{85,86}$. Visando preparar análogos do ácido helmintospórico (86), oxigenados na posição-8, Barbosa et alii ${ }^{24}$ e Demuner ${ }^{87}$ utilizaram a metodologia de cicloadição [3+4] entre cátions oxialílicos e furanos funcionalizados. No esquema 31 está apresentada a estratégia proposta para o preparo do ácido 93, a partir do cicloaduto 88. De acordo com esta proposta a conversão do oxabiciclo (91) ao produto desejado (93) envolveria a formação da $\beta$-lactama 92. Entretanto, o tratamento do alqueno 91 com clorossulfonilisocianato (CSI) resultou apenas na obtenção do monoterpeno 3-hidroxifelandral $(\mathbf{9 4})^{24}$.
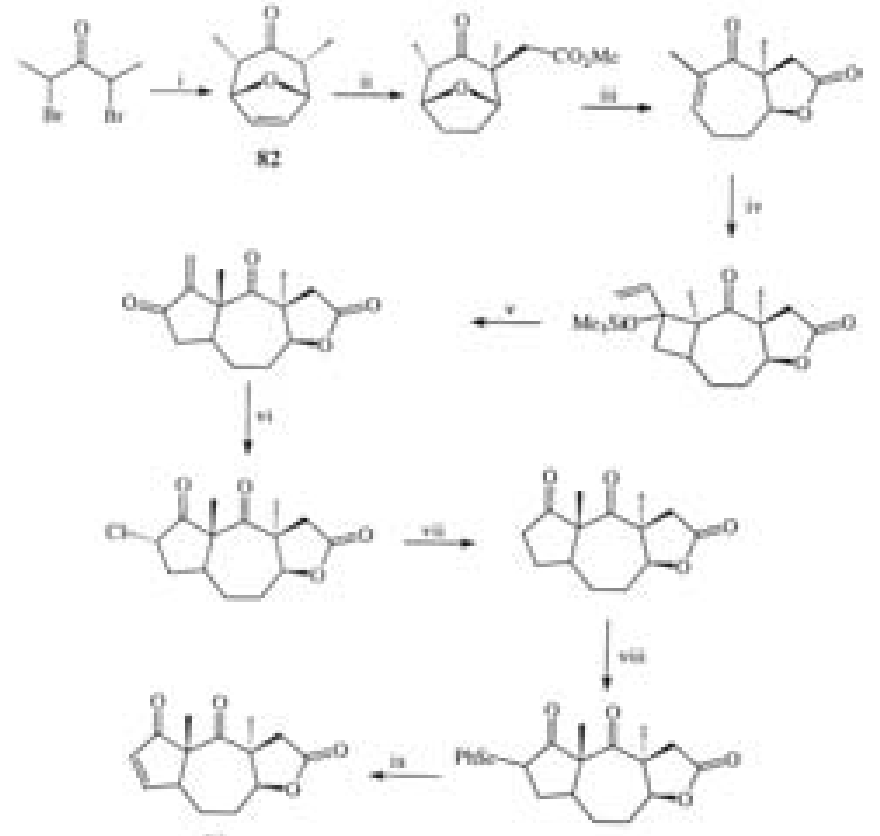

83

i) $\mathrm{Nal}, \mathrm{Cu}$, furano, $60 \%$; ii) $\mathrm{H}_{2}, \mathrm{Pd} / \mathrm{C}$; $\mathrm{LDA}, \mathrm{BrCH}_{2} \mathrm{CO}_{2} \mathrm{Me}, 96 \%$; iii) $\mathrm{BF}_{3}$, $\mathrm{KI}, 51 \%$; iv) $\mathrm{CH}_{2}=\mathrm{CHC}\left(\mathrm{OSiMe}_{3}\right)=\mathrm{CH}_{2}, \mathrm{hv}, 41 \%$; v) $\mathrm{Pd}(\mathrm{phCN})_{2} \mathrm{Cl}_{2}$, p-benzoquinona, 98\%; vi) $\mathrm{NabH}_{4} ; \mathrm{CCl}_{4} / \mathrm{Ph}_{3} \mathrm{P} ; \mathrm{NaIO}_{4}, \mathrm{OsO}_{4}, 62 \%$; vii) $\mathrm{Bu}_{3} \mathrm{SnH}, \mathrm{AIBN}, 83 \%$; viii) $\mathrm{PhSeCl}, 77 \%$; ix) $\mathrm{NaIO}_{4}, 25 \%$

Esquema 30
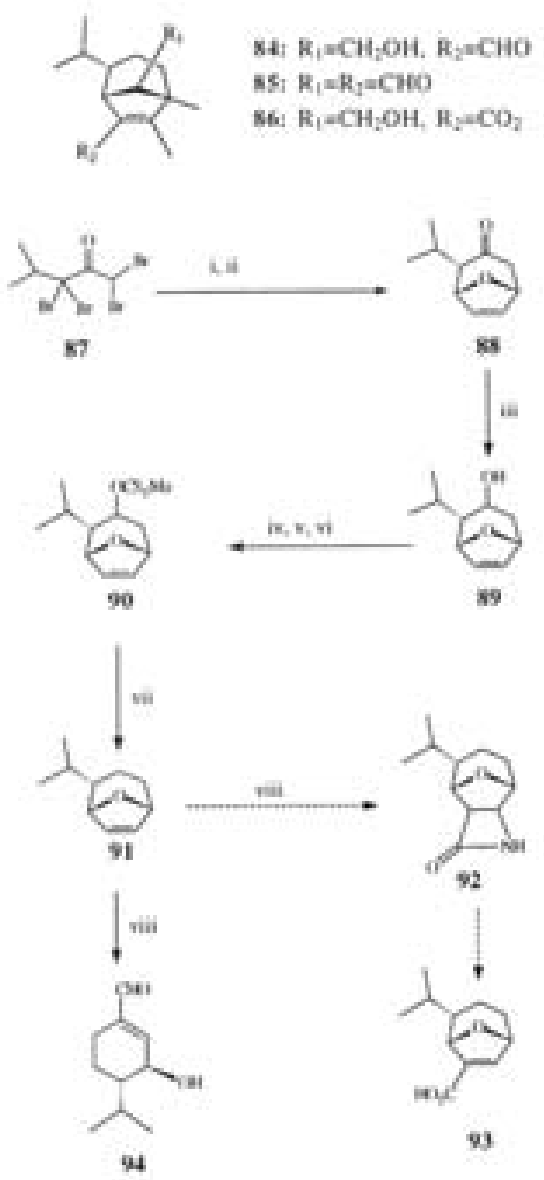

i) $\mathrm{Et}_{2} \mathrm{Zn}$, furano,benzeno; ii) $\mathrm{Zn} / \mathrm{Cu}, \mathrm{MeOH} / \mathrm{NH}_{4} \mathrm{Cl}, 55 \%$;

iii) DIBAH, $\mathrm{CH}_{2} \mathrm{Cl}_{2}, 95 \%$; iv) $\mathrm{NaH}$, imidazol; v) $\mathrm{CS}_{2}$; vi) $\mathrm{MeI}, 80 \%$; vii) $\mathrm{TBTH}, \mathrm{AIBN}, 95 \%$; viii) CSI, $20 \%$.

Esquema 31 
No esquema 32 está mostrada uma série de compostos obtidos através da cicloadição entre cátions oxialílicos e furanos.

Os compostos 88, 90, 91, 97-100, quando submetidos a testes de atividade herbicida, causaram $33-56 \%$ de inibição do sistema radicular do sorgo (Sorghum bicolor) ${ }^{87}$.

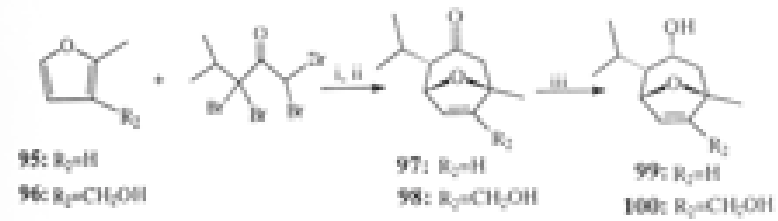

i) $\mathrm{Et}_{2} \mathrm{Zn}$, benzeno, $25^{\circ} \mathrm{C}, 30 \mathrm{~h}$; ii) $\mathrm{Zn} / \mathrm{Cu}, \mathrm{NH}_{4} \mathrm{Cl} / \mathrm{MeOH}, 25^{\circ} \mathrm{C}, 3 \mathrm{~h}$; (82: 54\%, 83: 57\%; iii) $\mathrm{NaBH}_{4} / \mathrm{MeOH}, 48$ h, (99: 98\%, 100: 90\%)

\section{Esquema 32}

\subsection{Obtenção de C-nucleosídeos}

O acetonídeo $\mathbf{1 0 1}$ foi convertido na lactona $\mathbf{1 0 2}$ por tratamento com ácido trifluoroperacético em $92 \%$ de rendimento ${ }^{39,40}$. Essa lactona foi, então, convertida em uma série de Cnucleosídeos (105-108) naturais e não-naturais por Noyori et $a l i i^{39,88}$ através dos intermediários $\mathbf{1 0 3}$ e 104 (Esquema 33).

Um grande número de homo-C-nucleosídeos, como por exemplo a homoshowdomicina (109), foi preparado por extensão da cadeia hidroxiéster (103), conforme mostrado no esquema $34^{40}$.

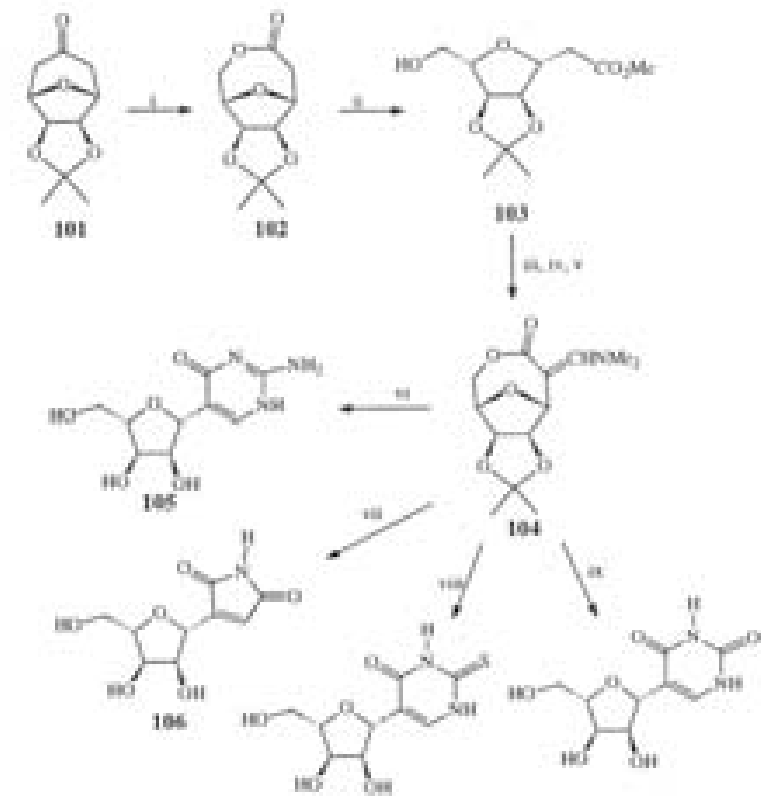

i) $\mathrm{CF}_{3} \mathrm{CO} 3 \mathrm{H}, \mathrm{CH}_{2} \mathrm{Cl}_{2}, 92 \%$; ii) $\mathrm{NaOMe}, \mathrm{MeOH}, 95 \%$;

iii) (R)-1-(1-naftil)etilisocianato, tolueno, $86 \%$; iv)Et ${ }_{3} \mathrm{~N}, \mathrm{Cl}_{3} \mathrm{SiH}, 80 \%$; v) t-BuOCH$\left(\mathrm{NMe}_{2}\right)_{2}$, DMF, 91\%; vi) guanidina. $\mathrm{HCl}, \mathrm{NaOEt}, \mathrm{HCl}, 72 \%$; vii) $\mathrm{O}_{3}, \mathrm{Me}_{2} \mathrm{~S}, \mathrm{Ph}_{3} \mathrm{PCHCONH}$, TFA, $72 \%$; viii) tiuréia, $\mathrm{NaOEt}, \mathrm{HCl}, 72 \%$; ix) uréia, $\mathrm{NaOEt}, \mathrm{HCl}, 55 \%$

\section{Esquema 33}

\subsection{Estudos visando o preparo do esqueleto taxano}

Uma das mais recentes aplicações da metodologia de cicloadição [3+4] envolvendo cátions oxialílicos corresponde ao trabalho de Oh et alii ${ }^{52}$, sobre o desenvolvimento de uma rota sintética para o esqueleto taxano (110).
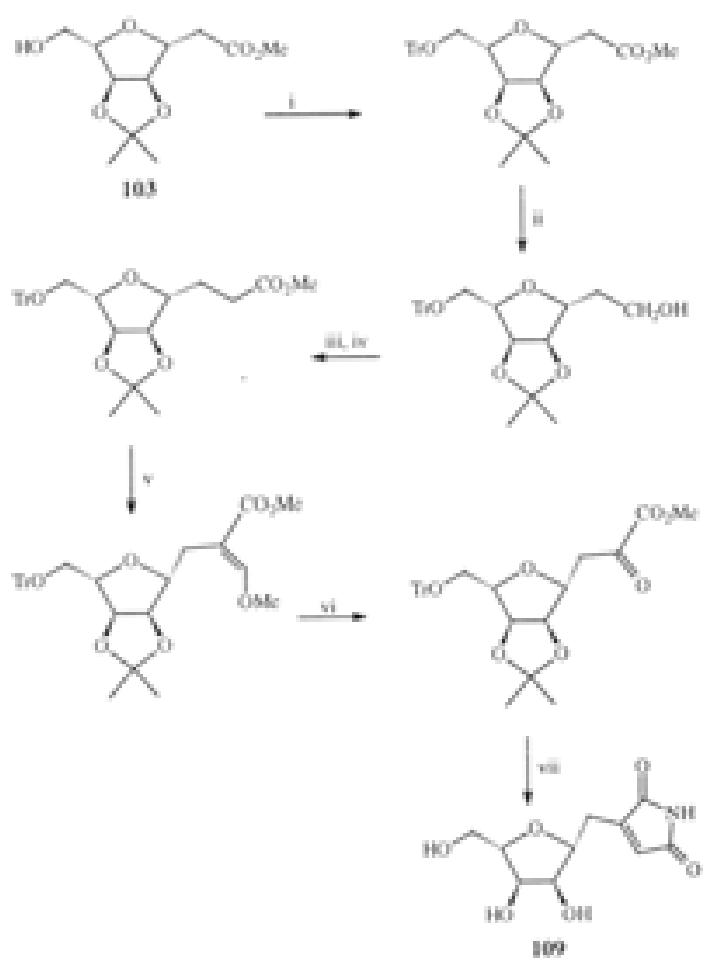

i) $\mathrm{Ph}_{3} \mathrm{CCl}, 100 \%$; ii) $\mathrm{LiAlH}_{4}, \mathrm{THF}, 83 \%$;

iii) piridina, $\mathrm{Tos} \mathrm{Cl}$; iv) $\mathrm{KCN}, \mathrm{KOH}, \mathrm{CH}{ }_{2} \mathrm{~N}_{2}, 30 \%$;

v) $\mathrm{LDA}, \mathrm{HCO}_{2} \mathrm{Me}, \mathrm{MeI}, 90 \%$; vi) $\mathrm{O} 3, \mathrm{Me}_{2} \mathrm{~S}, 100 \%$;

vii) $\mathrm{Ph}_{3} \mathrm{PCHCONH}$, TFA, 66\%

\section{Esquema 34}

Esta estrutura básica está presente no taxol (111) e vários outros diterpenos isolados de várias espécies do gênero Taxus. $\mathrm{O}$ grande interesse na síntese desses compostos deve-se às suas atividades anti-tumorais ${ }^{89-91}$.
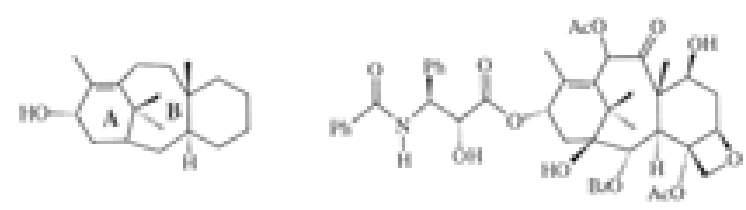

O trabalho de $\mathrm{Oh}$ et alii $^{52}$, apresentado resumidamente no esquema 35, teve como etapa chave a cicloadição [3+4] entre o dieno 112 e 3-cloro-2-pirrolidinocicloexeno (113), na presença de $\mathrm{AgBF}_{4}$. O cicloaduto 114 foi obtido com $42 \%$ de rendimento. Esta cetona 114 foi, então, por meio de uma sequência de reações, convertida nos compostos (115a e 115b), os quais possuem os anéis $\mathrm{AB}$ do esqueleto taxano funcionalizados adequadamente, de modo a permitir transformações posteriores para o preparo de análogos do taxol.
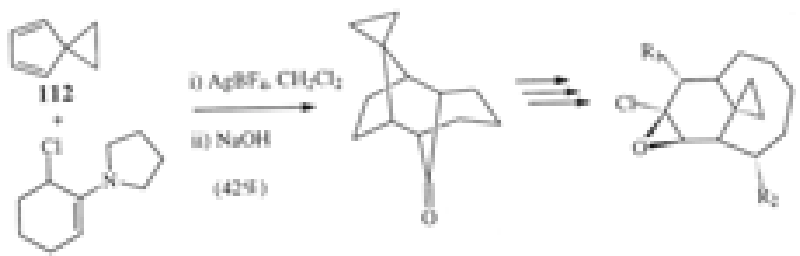

Esquema 35 


\section{CONCLUSÃ̃O}

Como ilustrado na série de reações apresentadas nesta revisão, os cátions oxialílicos constituem intermediários de síntese dos mais interessantes, por propiciarem a obtenção de substâncias de estruturas as mais versáteis, quer a partir dos produtos de suas reações de cicloadição, quer a partir das transformações químicas dos adutos cíclicos que originam.

\section{REFERÊNCIAS}

1. Buckingham, J.; Ed.; Dictionary of Natural Products. Chapaman \& Hall; London, 1994.

2. Hoffmann, H. M.; Angew. Chem. Int. Ed. Engl. 1973, 12, 819.

3. Fort, A. W.; J. Am. Chem. Soc. 1962, 84, 2620.

4. Fort, A. W.; J. Am. Chem. Soc. 1962, 84, 2625.

5. Fort, A. W.; J. Am. Chem. Soc. 1962, 84, 4979.

6. Cookson, R. C.; Nye, M. J.; J. Chem. Soc. 1965, 2009.

7. Cookson, R. C.; Nye, M. J.; Subrahmanyam, G.; J. Chem. Soc. (C). 1967, 473.

8. Mann, J.; Tetrahedron 1986, 42, 4611.

9. Hoffmann, H. M. R.; Angew. Chem. Int. Ed. Engl. 1984, 23,1 .

10. Noyori, R.; Acc. Chem. Res. 1979, 12, 61.

11. Noyori, R.; Hayakawa, Y.; Org. React. 1983, 29, 163.

12. Mann. J.; Usmani, A. A.; J. Chem. Soc. Chem Comm. 1980, 1119.

13. Mann, J.; Wilde, P. D.; Finch, M. W.; J. Chem. Soc. Chem. Comm. 1985, 1543.

14. Hosomi, A.; Tominaga, Y.; In Comprehensive Organic Synthesis; Trost, B. M., Ed.; Academic Press; London, 1991; p 593-615.

15. Föhlisch, B.; Gehriach, E.; Geywitz, B.; Chem. Ber. 1987, $120,1815$.

16. Föhlisch, B.; Flogaus, R.; Oexle, J.; Schadel, A.; Tetrahedron Lett. 1984, 25, 1773.

17. Harmata, M.; Elahmad, S.; Tetrahedron Lett., 1993, 34, 789.

18. Hoffmann, H. M. R.; Clemens, K. E.; Smithers, R. H.; $J$. Am. Chem. Soc. 1972, 94, 3940.

19. Sato, T.; Noyori, R.; Bull. Chem. Soc. Jpn. 1978, 51, 2745.

20. Noyori, R.; Hayamawa, Y.; Funakura, M.; Takaya, H.; Murai, S.; Kobayashi, R.; Tsutsumi, S.; J. Am. Chem. Soc. 1972, 94, 7202

21. Narula, A. S.; Tetrahedron Lett. 1979, 21, 1921.

22. Ishizu, T.; Mori, M.; Kanematsu, K.; J. Org. Chem. 1981, $46,526$.

23. Mann, J.; Barbosa, L. C. A.; J. Chem. Soc. Perkin Trans. 1. $1992,787$.

24. Barbosa, L. C. A.; Demuner, A. J.; Mann, J.; Piló-Veloso, D.; J. Chem. Soc. Perkin Trans. 1. 1993, 585.

25. Barbosa, L. C. A.; Mann, J.; Synthesis 1996, 31.

26. Hoffmann, H. M. R.; Iqbal, M. N.; Tetrahedron Lett. 1975, 50, 4487.

27. Ashcroft, M. R.; Hoffmann, H. M. R.; Organic Synthesis 1978, 58, 17.

28. Mann, J.; Holland, H. J.; Tetrahedron 1987, 43, 2533.

29. Hoffmann, H. M. R.; Weber, A.; Giguere, R. J.; Chem. Ber. 1984, 117, 3325.

30. Joshi, N. N.; Hoffmann, H. M. R.; Tetrahedron Lett. 1986 , 27, 687.

31. Föhlisch, B.; Gottstein, W.; Herter, R.; Wanner, T.; J. Chem. Res. (S). 1981, 246.

32. Bowers, K. G.; Mann, J.; Markson, A. J.; J. Chem. Res. (S). 1986, 424.

33. Olah, G. A.; Narang, S. C.; Gupta, B. G. B.; Malhotra, R.; Angew. Chem. Int. Ed. Engl. 1979, 18, 612.
34. Schmidt, G. A.; Aldrichimica Acta 1981, 14, 31

35. Maercker, A.; Angew. Chem. Int. Ed. Engl. 1987, 26, 972.

36. Barbosa, L. C. A.; Piló-Veloso, D.; Quím. Nova 1994, 17, 68.

37. Barbosa, L. C. A.; PhD Thesis; Reading University; Reading, 1991.

38. Noyori, R.; Kobayashi, R.; Sato, T.; Tetrahedron Lett. 1980, 21, 2573.

39. Noyori, R.; Sato, T.; Kobayashi, R.; Tetrahedron Lett. 1980, 21, 2569.

40. Sato, T.; Hayakawa, Y.; Noyori, R.; Bull. Chem. Soc. Jpn. 1984, 57, 2515

41. Hoffmann, H. M. R.; Joy, D.R.; J. Chem. Soc. (B) $\mathbf{1 9 6 8 ,}$ 1182.

42. Shimizu, N.; Tsuno, Y.; Chem. Lett. 1979, 103.

43. Shimizu, N.; Tanaka, M.; Tsuno, Y.; J. Am. Chem. Soc. 1982, 104, 1330.

44. Ohno, M.; Mori, K.; Hattori, T.; Eguchi, S.; J. Org. Chem. 1990, 55, 6086 .

45. Hoffmann, H. M. R.; Eggert, U.; Gibbels, U.; Giesel, K.; Koch, O.; Lies, R.; Rabe, J.; Tetrahedron 1988, 44, 3899.

46. Harmata, M.; Gamlath, C.B.; J. Org. Chem. 1988, 53, 6154.

47. Harmata, M.; Elahmad, S.; Barnes, C. L.; J. Org. Chem. 1994, 59, 1241.

48. Harmata, M.; Herron, B. F.; J. Org. Chem. 1993, 58, 7393.

49. Harmata, M.; Gamlath, C. B.; Barnes, C.L.; Tetrahedron Lett. 1990, 31, 5981.

50. Harmata, M.; Fletcher, V. R.; Classen, R. J.; J. Am. Chem. Soc. 1991, 113, 9861 .

51. Harmata, M.; Herron, B. F.; Tetrahedron Lett. 1993, 34, 5381.

52. Oh, J.; Choi, J. R.; Cha, J. K.; J. Org. Chem. 1992, 57 , 6664.

53. Harmata, M.; Gamlath, C. B.; Barnes, C. L.; Jones, D. E.; J. Org. Chem. 1995, 60, 5077.

54. Hoffmann, H. M. R.; Henning, R.; Lalko, O. R.; Angew. Chem. Int. Ed. Engl. 1982, 21, 442.

55. Barbosa, L. C. A.; Mann, J.; Wilde, P. D.; Finch, M. W.; Tetrahedron 1989, 45, 4619.

56. Barbosa, L. C. A.; Mann, J.; J. Chem. Soc. Perkin Trans. 1. 1990, 177.

57. Barbosa, L. C. A.; Cutler, D.; Mann, J.; Kirby, G. G.; Warhurst, D. C.; J. Chem. Soc. Perkin Trans. 1. 1992, 3253.

58. Barbosa, L. C. A.; Mann, J.; J. Chem. Soc. Perkin Trans. 1. $1992,337$.

59. Barbosa, L. C. A.; Mann, J.; Cummins, W. J.; J. Chem. Soc. Perkin Trans. 1. 1990, 3081.

60. Barbosa, L. C. A.; Mann, J.; Livro de Resumos do $6^{0}$ Encontro Regional da SBQ - MG, São João del Rei, 1992, 22.

61. Barbosa, L. C. A.; Cutler, D.; Mann, J.; Crabbe, M. J.; Kirby, G.G.; Warhurst, D. C.; J. Chem. Soc. Perkin Trans. 1. 1996, 1101.

62. Mak, C. P.; Buchi, G.; J. Org. Chem. 1981, 46, 1.

63. Sampath, V.; Schore, N. E.; J. Org. Chem. 1983, 48, 4882. 64. Föhlisch, B.; Herrscher, O.; Tetrahedron 1985, 41, 1979.

65. Takaya, H.; Hayakawa, Y.; Makino, S.; Noyori, R. J. Am. Chem. Soc. 1978, 100, 1778.

66. Klayman, D. L.; Science 1985, 228, 1049.

67. Zhan, W. S.; Xu, X.X.; In Studies in natural products chemistry; Atta-Ur Rahman, Ed.; Vol. 3, Elsevier; Amsterdam, 1989; p 287-293.

68. Butler, A. R.; Wu, Y. L.; Chem. Soc. Rev. 1992, 21, 85.

69. Cowling, A. P.; Mann, J.; J. Chem. Soc. Perkin Trans. 1. 1978, 1564.

70. Noyori, R.; Hayakawa, Y.; Tetrahedron 1985, 41, 5879.

71. Hayakawa, Y.; Baba, Y.; Makino, S.; Noyori, R.; J. Am. Chem. Soc. 1978, 100, 1786.

72. Hall, I. H.; Lee, K. H.; Starmes, C. O.; Sumida, Y.; Wu, 
R. Y.; Waddell, T. G.; Cochron, J. W.; Gerhart, K. G.; J. Pharm. Sci. 1979, 68, 537.

73. Hall, I. H.; Lee, K. C.; Sykes, H. C.; Planta Medica 1987, 2, 121

74. Cassady, J. M.; Suffness, M.; In Anticancer agents based on natural product models, Cassady, J. M.; Douxes, J. D.; Ed.; Vol. 16, Academic Press; New York, 1980; p 68-76.

75. Rodrigues, E.; Towers, G. H. N.; Mitchell, J. C.; Phytochemistry 1976, 15, 1573.

76. Picman, A. K.; Picman, J.; Biochem. Syst. Ecol. 1984, 12, 89.

77. Harmatha, J.; Nawrot, J.; Biochem. Syst. Ecol. 1984, 12, 95.

78. Armason, J. T.; Isman, M. B.; Philogene, J. R.; Waddell, T.G.; J. Nat. Prod. 1987, 50, 690.

79. Nawrot, J.; Harmatha, J.; Novotiny, L.; Biochem. Syst. Ecol. 1984, 12, 99.

80. Gennari, M.; Gentile, I. A.; Cugudda, L.; J. Plant Diseases Protection 1987, 94, 68.
81. Kupchen, S. M.; Fessler, D. C.; Eakin, M. A.; Giacobbe, T. J.; Science 1970, 168, 376.

82. Hanson, R. L.; Lardy, H. A.; Science 1970, 168, 373.

83. Talaya, P.; Schaeffer, M.; Mattes, H.; Benezra, C.; Stampt, J. L.; Tetrahedron 1989, 45, 5029.

84. Kupchan, S. M.; Eakin, M. A.; Thomas, A. M.; J. Med. Chem. 1971, 14, 1147.

85. Tamura, S.; Sakarai, A.; Agric. Biol. Chem. 1964, 337.

86. De Maio, P.; Spencer, E. Y.; White, R. W.; Can. J. Chem. 1961, 39, 1608.

87. Demuner, A. J.; Tese de Doutorado; Universidade Federal de Minas Gerais; Belo Horizonte, 1996.

88. Sato, T.; Watanabe, M.; Kobayashi, H.; Noyori, R.; Bull. Chem. Soc. Jpn. 1983, 56, 2680.

89. Denis, J. N.; Correa, A.; Greene, L. A. E.; J. Org. Chem. 1991, 56, 6939.

90. Borman, S.; Chem. Eng. News 1991, 2, 11.

91. Correa, A. G.; Quím. Nova 1995, 18, 460. 